\title{
UCN Source at an External Beam of Thermal Neutrons
}

\author{
E. V. Lychagin, ${ }^{1}$ A. Yu. Muzychka, ${ }^{1}$ G. V. Nekhaev, ${ }^{1}$ V. V. Nesvizhevsky, ${ }^{2}$ \\ E. I. Sharapov, ${ }^{1}$ and A. V. Strelkov ${ }^{1}$ \\ ${ }^{1}$ Joint Institute for Nuclear Research, 6 Joliot-Curie, Ru-141980, Dubna, Russia \\ ${ }^{2}$ Institute Max von Laue-Paul Langevin, 71 avenue des Martyrs, F-38000, Grenoble, France
}

Correspondence should be addressed to A. Yu. Muzychka; muz@nf.jinr.ru

Received 19 June 2014; Accepted 18 August 2014

Academic Editor: Stefan Baessler

Copyright (C) 2015 E. V. Lychagin et al. This is an open access article distributed under the Creative Commons Attribution License, which permits unrestricted use, distribution, and reproduction in any medium, provided the original work is properly cited. The publication of this article was funded by $\mathrm{SCOAP}^{3}$.

\begin{abstract}
We propose a new method for production of ultracold neutrons (UCNs) in superfluid helium. The principal idea consists in installing a helium UCN source into an external beam of thermal or cold neutrons and in surrounding this source with a solid methane moderator/reflector cooled down to $\sim 4 \mathrm{~K}$. The moderator plays the role of an external source of cold neutrons needed to produce UCNs. The flux of accumulated neutrons could exceed the flux of incident neutrons due to their numerous reflections from methane; also the source size could be significantly larger than the incident beam diameter. We provide preliminary calculations of cooling of neutrons. These calculations show that such a source being installed at an intense source of thermal or cold neutrons like the ILL or PIK reactor or the ESS spallation source could provide the UCN density $10^{5} \mathrm{~cm}^{-3}$, the production rate $10^{7} \mathrm{UCN} / \mathrm{s}^{-1}$. Main advantages of such an UCN source include its low radiative and thermal load, relatively low cost, and convenient accessibility for any maintenance. We have carried out an experiment on cooling of thermal neutrons in a methane cavity. The data confirm the results of our calculations of the spectrum and flux of neutrons in the methane cavity.
\end{abstract}

\section{Introduction}

Further progress in the field of studies with ultracold neutrons (UCNs) [1,2], as a tool for nuclear and particle physics [3-5], is often limited by available UCN densities. Therefore many projects of new advanced UCN sources are pursued in various scientific centers in the world; they are aiming to increase the available UCN densities and fluxes by at least 1-2 orders of magnitude.

The principle of production of UCNs in superfluid helium was proposed already in 1975 in [6]. It is based on the fact that a neutron could excite a phonon with the energy of $1.02 \mathrm{meV}$ in liquid ${ }^{4} \mathrm{He}$. If the energy of incident neutron exceeds slightly this value $(1.02 \mathrm{meV})$, the neutron will be scattered to the UCN energy. UCNs could be produced via this mechanism only from incident cold neutrons with energies from a very narrow range as the UCN energy is as small as $<300 \mathrm{neV}$. A neutron could also excite two or more phonons in helium simultaneously. The cross section of such multiphonon scattering is lower by several orders of magnitude than the cross section of one-phonon scattering. However the total energy of exited phonons could be found within a much broader energy range, and thus UCNs could be produced in a multiphonon process from a much broader energy range of incident neutrons. Therefore both processes would give comparable contributions to the UCN production provided that the initial cold neutron spectrum is broad.

It was also shown in the cited work that the produced UCNs could be stored in superfluid helium for a long time if the helium temperature is $\sim 1 \mathrm{~K}$ or lower. This fact allows building up the UCN density in a source of this type up to very high values.

Helium UCN sources, as well as solid-deuterium UCN sources, seem to be most promising at present. There are several projects of helium UCN sources in the stage of their realization or design [7-11]. A drawback of helium sources as compared to solid-deuterium sources consists of very low temperatures needed in order to provide long UCN lifetimes in superfluid ${ }^{4} \mathrm{He}$ : thus the lifetime drops down from $10^{4} \mathrm{~s}$ to $1 \mathrm{~s}$ if the temperature increases from $0.6 \mathrm{~K}$ to $2 \mathrm{~K}[12,13]$. 
Advantages of helium sources consist of the transparency of the physical processes involved, the relatively low cost, and the possibility to install them outside of a reactor/spallation source zone.

\section{The Concept of UCN Sources at External Beams of Thermal Neutrons}

It was noted in [14] in 1998 that if an UCN source filled in with liquid helium at the temperature of below $1 \mathrm{~K}$ is installed in a beam of cold neutrons surrounded with a cold neutron reflector (i.e., the reflector provides a trap for cold neutrons used to produce UCNs), then one could noticeably increase the flux of cold neutrons in the source and thus increase the UCN generation rate. Beryllium oxide and Plexiglas at a temperature of several degrees were considered as possible materials for the reflector. The mean path of cold neutrons in the source of a cylindrical or spherical shape could increase by the factors 2.2 and 2.5 accordingly. This idea has not been realized as such gains could be achieved only in relatively small volumes. The reason is in the fact that the angular distribution of reflected neutrons is isotropic in contrast to the initial narrow angular distribution. Thus one could not use a section of a neutron guide (with the length several times larger than the width) for such a source; the path of cold neutrons in a guideshaped source (and thus the generation rate) would be higher anyway than in a spherical source of equal volume.

However if we replace the reflector material by another one providing simultaneously good moderation and reflection properties, then one could (1) considerably increase the flux of cold neutrons in the source and thus its production rate and also (2) use "cheap" and intense thermal neutrons instead of "expensive" and less intense cold neutrons for UCN production [15]. In fact such a moderator/reflector plays the role of a cold neutron source. Thus the maximum flux density in beams of cold neutrons at the ILL reactor reaches $\sim 10^{10} \mathrm{~cm}^{-2} \mathrm{~s}^{-1}$, while the maximum flux density in beams of thermal neutrons at the same reactor could reach $\sim 10^{11}$ $\mathrm{cm}^{-2} \mathrm{~s}^{-1}$ as it will be shown in the next section.

Such an UCN source is shown schematically in Figure 1; it is surrounded with a moderator/reflector, which plays the role of an external cold neutron source. The moderator/ reflector in the figure surrounds also the input neutron guide in order to avoid leakages of neutrons (from the trap) with the angular divergence exceeding the narrow angular divergence in the initial neutron beam. The larger the albedo of the reflector is, the higher the saturated neutron flux density in the source is; in the limit of the reflector albedo equal unity, the neutron flux density accumulated in the cavity is strictly equal to the neutron flux density in the thermal neutron source. For the PIK and ILL reactors it would be equal to $\sim 10^{15} \mathrm{n} \cdot \mathrm{cm}^{-2} \mathrm{~s}^{-1}$.

A matter with maximum albedo for cold neutrons, which we have found, is solid methane in phase II at the temperature of $\sim 4 \mathrm{~K}$ (we leave aside composite materials based on nanoparticle reflectors [16-18], which we are analyzing currently in view of even further increase in the attainable albedo). On the one hand, albedo of methane is slightly

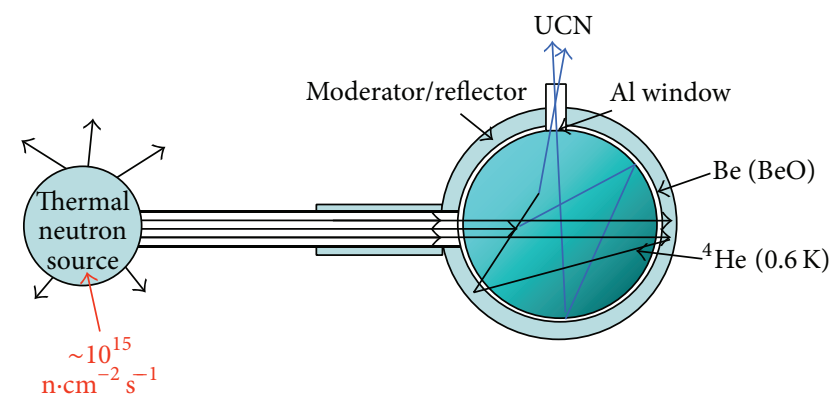

FIgURE 1: A scheme of an UCN source surrounded by a moderator/reflector.

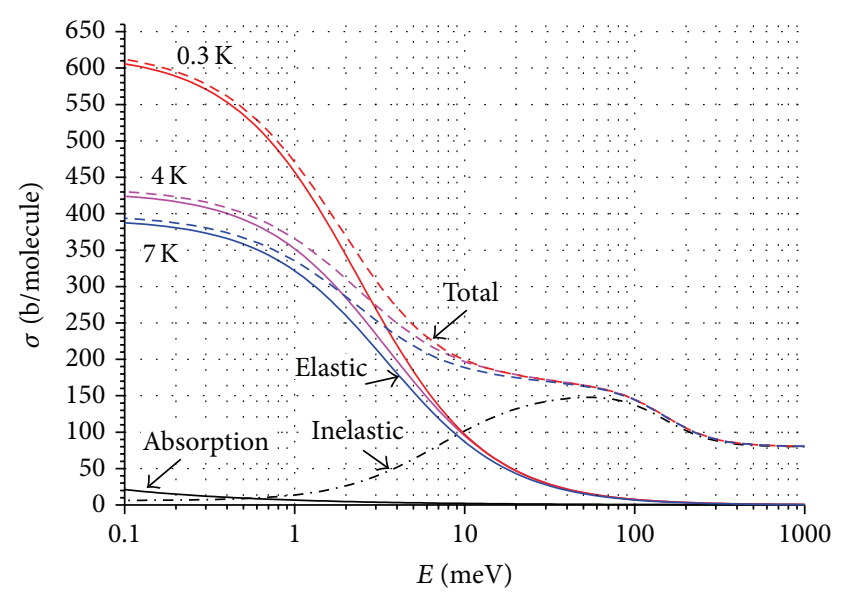

FIGURE 2: The cross section of neutron scattering normalized to one molecule of solid methane $\left(\mathrm{CH}_{4}\right)$ in the phase II at different temperatures is shown as a function of neutron energy.

higher than albedo of reflectors considered in [9]. On the other hand, solid methane is simultaneously one of the best cold moderators. This latter property of methane is due to the fact that it is a molecular crystal with a very round molecule; thus it provides rather flexible rotational degrees of freedom even at very low temperatures.

Another particularity of solid methane is parallel alignment of spins of hydrogen atoms in the methane molecule at low temperatures. The scattering of low energy neutrons becomes coherent for one molecule and stays incoherent for different molecules. Therefore there is no optical potential for neutrons of low energy as there is for materials with coherent scattering, and the cross section increases with decreasing the neutron energy. Cross sections of neutron scattering in solid methane at different temperatures are presented in Figure 2 as a function of neutron energy; the data are taken from $[19,20]$.

Solid color lines correspond to the cross sections of elastic scattering at respective temperatures. Black dashed-dotted line indicates the cross section of inelastic scattering. Color dashed lines show the total scattering cross section at a given temperature. Black solid line gives the absorption cross section. 


\section{Estimation of Thermal Neutron Flux Density in a Neutron Guide}

Before calculating parameters of the proposed UCN source, we estimate the flux of thermal neutrons, which could be achieved in an external neutron guide of existing neutron sources.

The most intense worldwide steady neutron source is currently the ILL reactor; it provides the thermal neutron flux density of $J_{0}=1.2 \cdot 10^{15} \mathrm{n} /\left(\mathrm{cm}^{2} \mathrm{~s}\right)$ in the vicinity of its active zone. The distance from its active zone to the outside surface of its biological shielding is about $4 \mathrm{~m}$. There are holes with the maximum diameter of $20 \mathrm{~cm}$ in the biological shielding of the reactor for the installation of neutron guides. It is realistic to install an UCN source at the end of a neutron guide with the diameter of $20 \mathrm{~cm}$ at the distance of $5 \mathrm{~m}$ from the center of the reactor active zone.

So, let us assume that there is a cylindrical tube with the length of $L=5 \mathrm{~m}$ and the diameter of $D=20 \mathrm{~cm}$ (the square area cross section is thus $S=314 \mathrm{~cm}^{2}$ ). Let us assume also that one end of the tube is an isotropic source of thermal neutrons with the flux density equal to $J_{0}=1.2 \cdot 10^{15} \mathrm{n} /\left(\mathrm{cm}^{2} \mathrm{~s}\right)$. Then the neutron flux density through the opposite end of the tube is

$$
J=J_{0} \frac{S}{4 \pi L^{2}}=J_{0} \cdot 10^{-4}=1.2 \cdot 10^{11} \mathrm{n} /\left(\mathrm{cm}^{2} \mathrm{~s}\right) .
$$

The integral flux will be equal to

$$
F=3.8 \cdot 10^{13} \mathrm{n} / \mathrm{s} .
$$

Note that a real neutron flux could be about twice larger due to the radiation of neutrons through side walls of the tube and reflection of neutrons from the biological shielding.

Note also that the flux density is proportional to the square of the tube diameter, and the integral flux is proportional to the 4 th power of the diameter.

\section{Modeling of Neutron Spectrum in the Solid Methane Cavity}

We have simulated the spectrum of neutrons accumulated in a solid methane cavity. The simulation was carried out using the program MCNP $4 c$ with a special kern for solid methane; the kern was used in $[20,21]$ and was kindly provided to us by the authors.

As a result of this model calculation, we established the optimum parameters of moderator/reflector. We found that the optimum thickness of methane is $\sim 3 \mathrm{~cm}$; an increase in its thickness does not increase noticeably the number of neutrons in the cavity. The optimum temperature of methane is $\sim 4 \mathrm{~K}$; a decrease in the temperature also does not increase the number of neutrons in the cavity. The optimum diameter of the cavity is $40-50 \mathrm{~cm}$ if the input neutron guide diameter is $20 \mathrm{~cm}$.

Figures 3 and 4 show spectra of neutrons accumulated in a spherical cavity with the internal diameter of $40 \mathrm{~cm}$ surrounded with solid methane. Here $\Phi$ is the neutron fluence averaged over the cavity volume per one incident neutron.

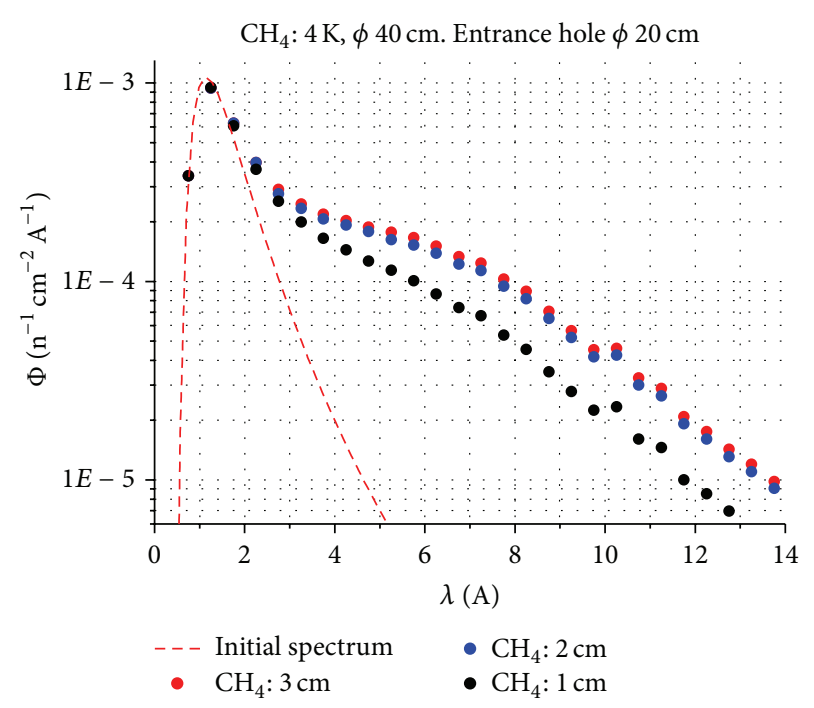

FIGURE 3: Mean neutron fluence in a spherical cavity with the diameter of $40 \mathrm{~cm}$ surrounded with solid methane at the temperature of $4 \mathrm{~K}$ is shown as a function of neutron wavelength.

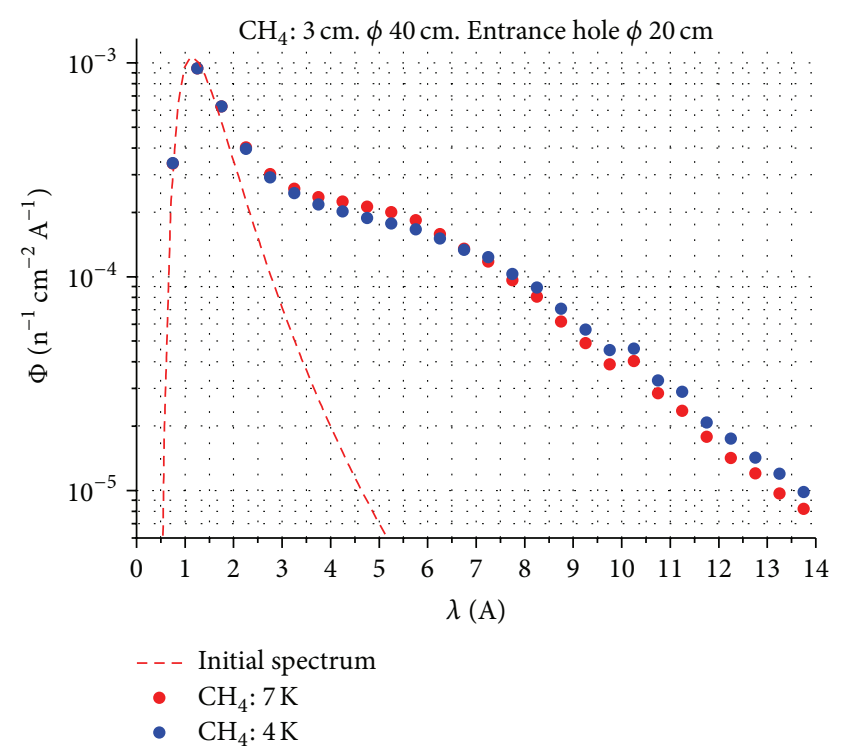

FIGURE 4: Mean fluence in a spherical cavity with the diameter of $40 \mathrm{~cm}$ surrounded with solid methane walls with the thickness of $3 \mathrm{~cm}$.

The velocity distribution of incident neutrons is Maxwellian with the temperature of $300 \mathrm{~K}$; they enter the cavity through the input guide with the diameter of $\phi 20 \mathrm{~cm}$.

Line indicates the mean fluence of incident neutrons with the temperature of $300 \mathrm{~K}$ normalized per 1 neutron.

Points show the mean fluence of accumulated neutrons, normalized per 1 incident neutron, for different thicknesses of the cavity walls equal to 1,2 , and $3 \mathrm{~cm}$.

Line indicates the mean fluence of incident neutrons with the temperature of $300 \mathrm{~K}$ normalized per 1 neutron. 
Points show the mean fluence of accumulated neutrons, normalized per 1 incident neutron, for different temperatures of methane equal to $7 \mathrm{~K}$ and $4 \mathrm{~K}$.

\section{Estimation of the Generation Rate in the Source}

For one-phonon processes only and for the UCN energy below the beryllium optical potential, the UCN generation rate in liquid helium [22] equals

$$
R_{1}=4.55 \cdot 10^{-8} \frac{d J}{d \lambda}(8.9 \AA) \mathrm{cm}^{-3} \mathrm{~s}^{-1} .
$$

For the input flux (2), a spherical cavity with the diameter of $40 \mathrm{~cm}$, and the mean fluence $d \Phi / d \lambda(8.9 \AA)=6.7$. $10^{-5} \mathrm{n} /\left(\mathrm{cm}^{2} \AA\right)$, (see Figures 3 and 4$)$ one could get

$$
\begin{gathered}
\frac{d J}{d \lambda}(8.9 \AA)=2.5 \cdot 10^{9} \mathrm{n} / \mathrm{cm}^{2} \mathrm{~s} \AA, \\
R_{1}=116 \mathrm{n} /\left(\mathrm{cm}^{3} \mathrm{~s}\right) .
\end{gathered}
$$

Estimations of UCN production due to multiphonon processes differ in different works. Thus for the spectra of accumulated neutrons as shown in Figures 3 and 4, multiphonon processes could contribute with a factor of increase in the UCN production rate equal to a value ranging from 1.25 to 3.5 [22-24]. Thus the lower estimation of the UCN production rate is $R=145 \mathrm{n} /\left(\mathrm{cm}^{3} \mathrm{~s}\right)$.

The total number of UCNs produced in the source per second is $5 \cdot 10^{6} \mathrm{UCN} / \mathrm{s}$.

One could propose the following characteristics of the source: the number of UCNs produced in the source per one incident thermal neutron is $1.3 \cdot 10^{-7} \mathrm{UCN} / \mathrm{s} / \mathrm{n}$.

For the coefficient of loss of UCNs in the walls of the UCN accumulation volume equal to $\eta=10^{-4}$, the mean partial storage time of UCNs related to wall losses is $\tau_{w} \approx 450 \mathrm{~s}$; with account for the neutron $\beta$-decay lifetime the storage time will be equal to $\tau \approx 300 \mathrm{~s}$.

Preliminary estimations indicate that the thermal heat to the source with the diameter of $40 \mathrm{~cm}$ will raise to $\sim 0.2 \mathrm{~W}$ for the input neutron flux (2); at such a heat load the source could be cooled down to the temperature of $0.6 \mathrm{~K}$.

If the helium temperature is $0.8 \mathrm{~K}$, the UCN storage time in helium is equal to the neutron $\beta$-decay lifetime. If the helium temperature is $0.6 \mathrm{~K}$, it is a factor of 10 larger and thus such losses even could be neglected.

Thus, the maximum UCN density in the source is

$$
n_{\mathrm{UCN}}=R \cdot \tau=4.4 \cdot 10^{4} \mathrm{UCN} / \mathrm{cm}^{3} .
$$

We have given the lower estimate for the UCN production rate in the source. The upper estimation would be a factor of $\sim 5$ larger.

These estimations are valid for a source with the diameter of $40 \mathrm{~cm}$ and the neutron guide diameter of $20 \mathrm{~cm}$. If the source diameter is $30 \mathrm{~cm}$, the UCN density would practically stay the same because the leakage of $8.9 \AA$ "parent neutrons" through the input neutron guide will increase. On the other

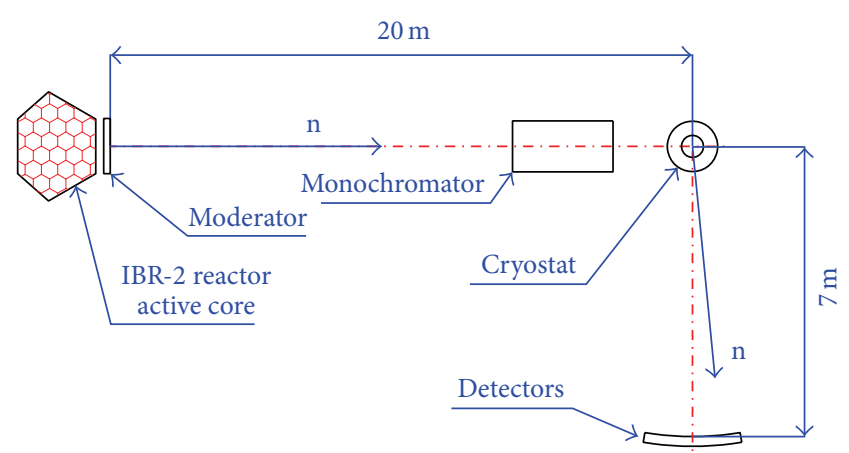

FIGURE 5: A scheme of measurement of the spectra and intensities of neutrons accumulated in a methane cavity performed with the instrument DIN-2Pi.

hand, the total number of produced UCNs ("children") would decrease by a factor of $\sim 2$. An increase of the source size will increase the production rate proportionally to the diameter value; however it will decrease the UCN density proportional to the reciprocal diameter square.

\section{Measurements}

In order to verify validity of the calculations presented in Figures 3 and 4, we have measured the spectra and intensities of neutrons accumulated in a solid methane cavity.

The measurements were performed using the instrument DIN-2Pi [25] at the reactor IBR-2 [26] (JINR, Dubna). The instrument was designed to measure inelastic scattering of neutrons using the time-of-flight method. The length of the input arm of DIN-2Pi is $20 \mathrm{~m}$; it is measured between the reactor and a sample, which scatters neutrons (a cryostat with solid methane in our case). The output arm length is $7 \mathrm{~m}$; it is measured between the sample and the detectors. Choppers are installed in the input arm of the spectrometer; they select a monoline with the wavelength (time-of-flight) resolution of $\sim 1 \%$.

A scheme of measurement is shown in Figure 5.

A sample of solid methane with a cylindrical cavity measuring $12 \times 12 \mathrm{~cm}$ with the wall thickness of $3 \mathrm{~cm}$ was grown in the cryostat. The temperature of methane was slightly different in different points of the sample within the range from $6 \mathrm{~K}$ to $7.2 \mathrm{~K}$. The temperature was stable in time. Two holes were left in the walls of methane cavity, one for the neutron entrance and another one for their exit, at the angle of $90^{\circ}$ relative to each other. The square area of every hole was equal to $\sim 7 \mathrm{~cm}^{2}$. The cryostat was surrounded with Cd-shielding so that thermal neutrons could enter into the cryostat only through the entrance window, and they could escape from the cavity to the detectors only through the exit window. Before escaping into the detector, neutrons had to have two or more reflections from the cavity walls.

\section{Results of the Measurements}

We selected only one monoline of incident thermal neutrons with the energy of $25.0 \mathrm{meV}$ (1.81 $\AA$ ). There were also many 


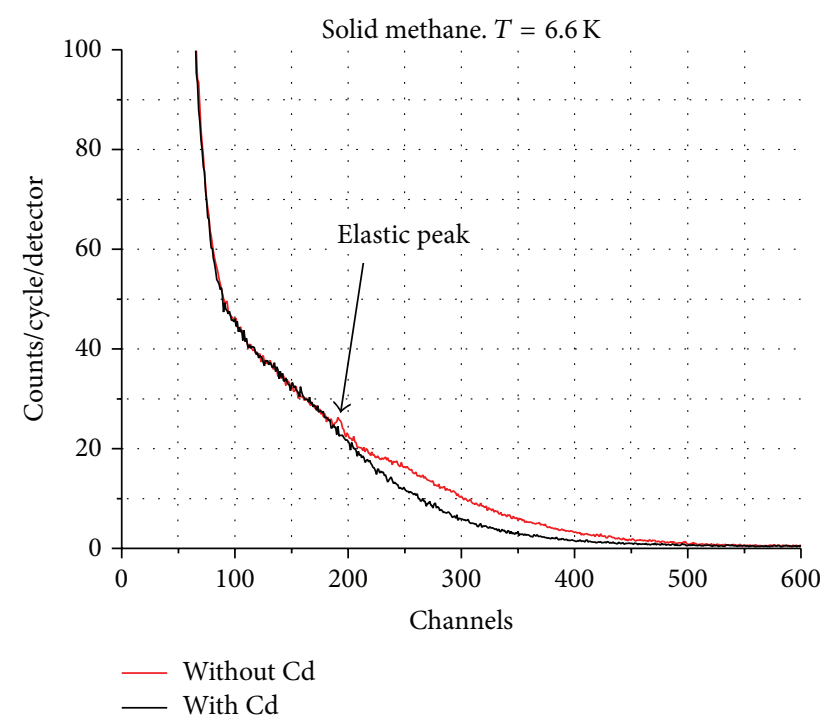

FIGURE 6: Count rate in the detector as a function of the time interval after a reactor pulse. Results of measurements with the initial beam open are shown with red line. Results of measurements with a sheet of cadmium in the incident neutron beam are indicated with black line. The width of one time channel is $64 \mu \mathrm{s}$.

"background" fast and epithermal neutrons (that could pass through a cadmium sheet) in the initial beam. A major fraction of these neutrons could be separated using the timeof-flight technique. However some faster neutrons could penetrate into the cryostat, scatter in methane, and escape to the detector during the same time intervals as neutrons from the initial thermal monoline do. To exclude an influence of fast neutrons, we measured the so-called cadmium difference, that is, the difference of the count rate with the input beam open and the count rate with a sheet of cadmium in the input beam, which totally absorbed the initial thermal beam. Figure 6 shows the results of these measurements.

The difference of these measurements allows calculating the spectrum of neutrons thermalized in methane.

Figure 7 presents the results of measurements of the spectrum of neutrons escaping from the methane cavity through the exit window. We performed a numerical simulation of our experiment using the same computer MCNP $4 \mathrm{c}$ code as used for the source simulation. The numerical simulation almost ideally coincides with the measurements, except for one feature, which is not important in this case. Namely, one clearly observes measured extra neutrons, which did not change their energy during their storage in the cavity, the elastic peak. We do not reproduce this peak in simulations.

Also we show Maxwellian spectra in Figure 7 for comparing them with the measured results. One spectrum (dashed line) provides the maximum number of neutrons with the wavelength of $8.9 \AA$ (our "ideal" spectrum). Another one (solid blue line) provides a shape closest to the measured data. This latter spectrum is also very close to cold neutron spectra in beams at the ILL. The integrals under all spectra are equal to each other.

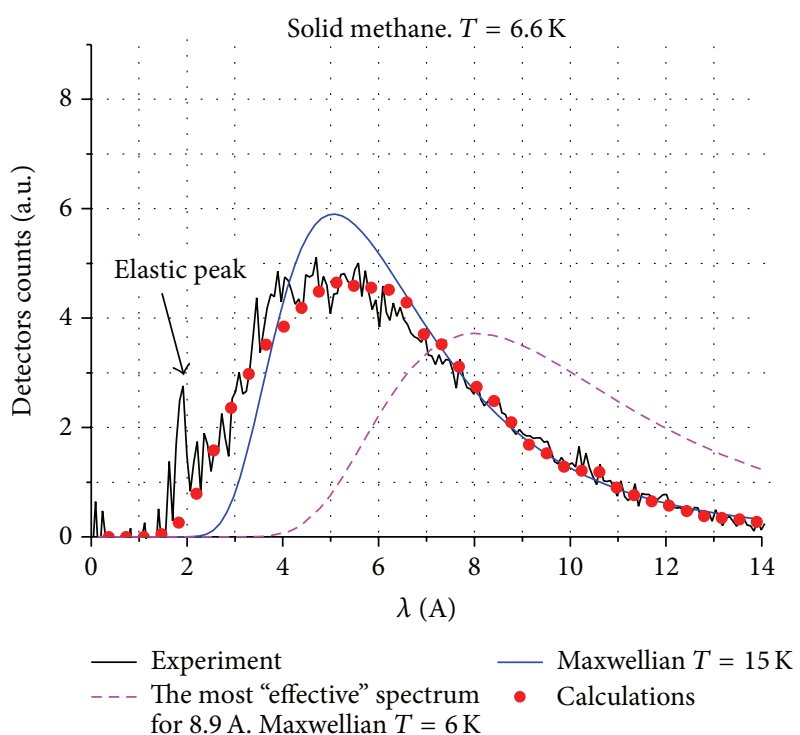

FIGURE 7: Spectrum of neutrons escaping from the methane cavity through the exit window. Thin line indicates the results of measurements. Points correspond to the numerical simulation of our experiment. Dashed line illustrates the Maxwellian distribution, which provides the largest count rate of neutrons with the wavelength of 8.9 A. Blue line shows a Maxwellian distribution fitting the data in the best way. The integrals under all curves are equal to each other.

As an additional verification of validity of simulations of neutron fluxes accumulated in the methane cavity, we performed an analogous measurement with methane replaced with distillated water at the temperature of $292 \mathrm{~K}$. In this test, the number of initial neutrons entering the cryostat was the same, and the spectrum was the same, as in measurements with methane. Results of this measurement are shown in Figure 8.

The initial spectrum, as mentioned above, is a monoline with the wavelength width smaller than $1 \%$. Such a width could not be shown graphically in the scale in Figure 8 (the "elastic peak" in Figures 6 and 7 has larger width as it is washed out due to time-of-flight spread caused by some delay of neutrons in the methane cavity).

As clear from this figure, the results of numerical simulations perfectly agree with the measured data. Also the ratios of integrals under the spectra for water and methane agree with results of simulations and measurements.

Experiment:

$$
\frac{\int N_{\operatorname{det}}^{\mathrm{H}_{2} \mathrm{O}}(\lambda) d \lambda}{\int N_{\operatorname{det}}^{\mathrm{CH}_{4}}(\lambda) d \lambda}=2.01 \pm 0.02 .
$$

Simulation:

$$
\frac{\int N_{\operatorname{det}}^{\mathrm{H}_{2} \mathrm{O}}(\lambda) d \lambda}{\int N_{\operatorname{det}}^{\mathrm{CH}_{4}}(\lambda) d \lambda}=1.94 \pm 0.04 .
$$

Here $N_{\text {det }}^{\mathrm{H}_{2} \mathrm{O}}$ is the count rate for water at the temperature of $292 \mathrm{~K}$ and $N_{\operatorname{det}}^{\mathrm{CH}_{4}}$ is the count rate for methane at the temperature of $6.6 \mathrm{~K}$. 


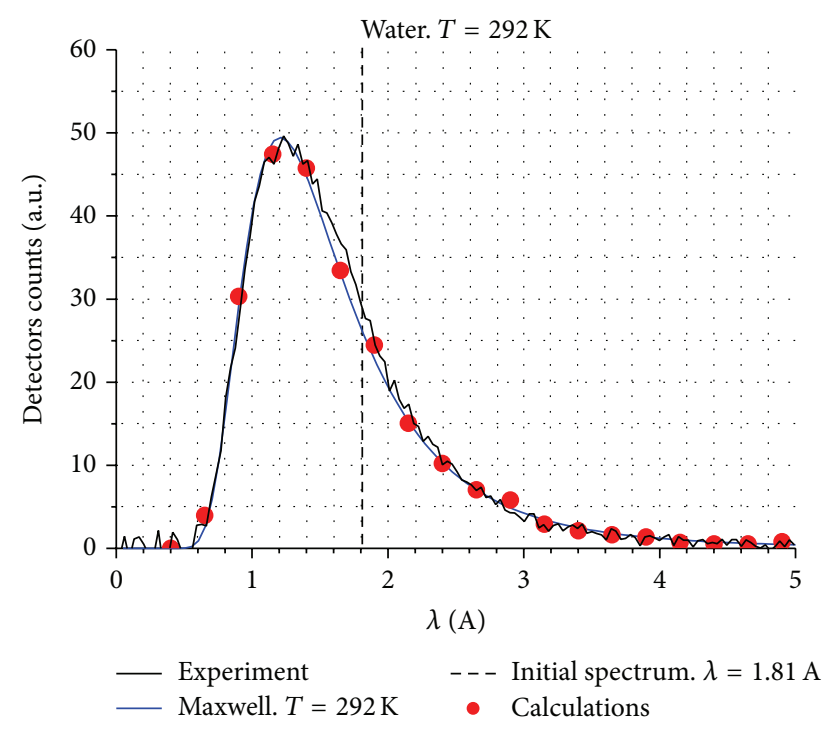

FIGURE 8: The spectrum of neutrons escaping from the water cavity through the exit window is shown as a function of neutron wavelength. Thin line indicates the measured results. Points correspond to the results of numerical simulations of this experiment. Blue line indicates the Maxwellian distribution for the temperature equal to $292 \mathrm{~K}$.

All these performed measurements demonstrated high accuracy of simulations both in terms of absolute values of neutron fluxes accumulated in a methane cavity and also in terms of spectra of accumulated neutrons. Accordingly, we confirm all simulated characteristics of the UCN source. On the other hand, one should underline that now we could provide rather precise estimations of these characteristics basing these estimations solely on the measured data, even without simulations.

In fact, if we know the value of albedo of thermal neutrons from water, then relation (6) and a known geometry of the cavity (see above) allow calculating the albedo from solid methane.

MCNP simulations of neutron albedo from water at the temperature of $292 \mathrm{~K}$, for normal incidence of neutrons with the energy of $25 \mathrm{meV}$, provide the value $0.787 \pm 0.008$. Nearly the same value could be obtained for isotropic incidence of neutrons with the Maxwellian distribution with the temperature of $292 \mathrm{~K}: 0.806 \pm 0.008$. Thus, in all cases typical for experiments, the albedo from water is about 0.8 . Taking into account that we observe in experiments the neutrons after their second and further reflections from the cavity walls and also taking into account the sizes of entrance and exit holes and relation (6), we get albedo from methane equal to 0.65 . Assuming the spectrum of neutrons in the methane cavity is the same as that shown in Figure 7, one could calculate the neutron flux in a methane cavity of any geometry and thus the UCN production rate in the source.

The characteristics of the UCN source estimated in this way are higher by $\sim 20 \%$ than those given above that means a very good accuracy. The main reason for this deviation consists of the fact that the neutron energy after their first

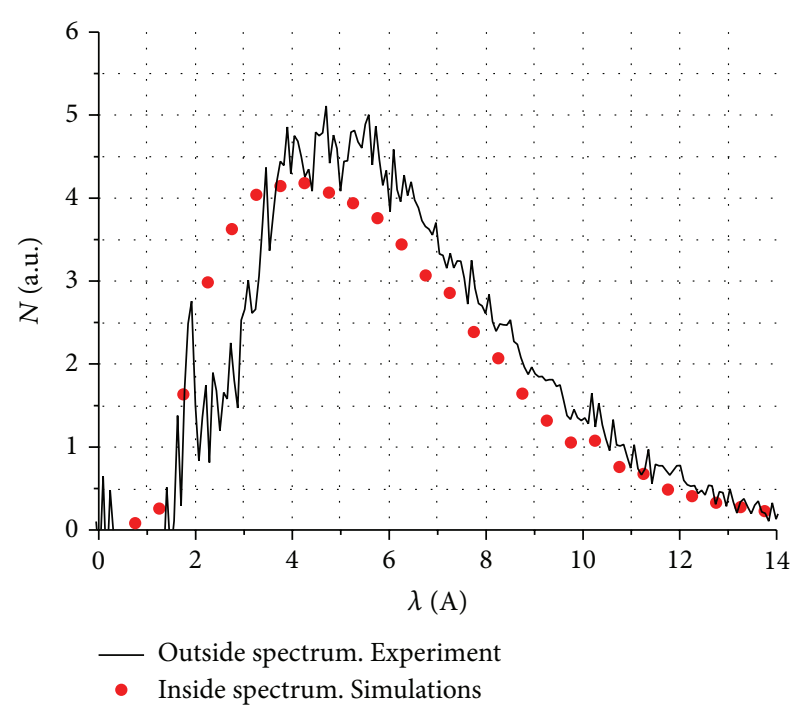

FIGURE 9: The neutron spectrum inside the methane cavity and the spectrum of neutrons escaping through the exit hole are compared. Thin line indicates results of measurements outside of the cavity. Points correspond to the numerical simulation of the spectrum inside the cavity.

reflection from a methane cavity wall is higher than their energy after subsequent reflections, and the contribution of such neutrons into the total neutron flux is more important (as the number of neutrons decreases after each reflection).

The measured spectrum (Figure 7) is compared with the simulated spectrum inside the cavity (Figures 3 and 4) in Figure 9. For convenience of normalization, we excluded from the simulated spectrum inside the cavity a contribution of neutrons from the initial beam; they marginally affect the UCN production.

\section{Conclusion}

Our calculations showed that the installation of a helium UCN source in an external beam of thermal neutrons at the ILL reactor in Grenoble, at the PIK reactor in Gatchina, or at the ESS spallation source in Lund would allow achieving the $\mathrm{UCN}$ density of $\sim 10^{5} \mathrm{UCN} \cdot \mathrm{cm}^{-3}$. The production rate of such an UCN source is $\sim 10^{7} \mathrm{UCN} \cdot \mathrm{s}^{-1}$. Our measurements confirmed validity of these calculations.

The main source of thermal load in such a source, if it is installed at a heavy-water reactor like that at the ILL, for instance, consists of gamma-quanta produced in the capture of neutrons in the moderator/reflector itself. The estimated value of the heat load caused by such gamma-quanta in helium of the UCN source is $0.2 \mathrm{~W}$ for the considered geometry and the neutron flux (2). This estimation of the heat load means that one could even considerably increase the flux of incoming neutrons, and thus one could increase the density and the flux of UCNs in the source. For instance, an increase of the diameter of the input neutron guide from $20 \mathrm{~cm}$ to $30 \mathrm{~cm}$ would increase the input neutron flux by a factor of $\sim 5$. However, one should take into account that a 
further increase of the heat load over 1-2 W would complicate cooling of liquid helium in the source below the required temperature of $0.8 \mathrm{~K}$ provided that the method of pumping of ${ }^{3} \mathrm{He}$ vapors or the method of dilution of ${ }^{3} \mathrm{He}$ in ${ }^{4} \mathrm{He}$ is used. Therefore the limit of production rate in such a source is probably close to $\sim 10^{8} \mathrm{n} \cdot \mathrm{s}^{-1}$.

On the one hand, the proposed UCN source provides parameters much better than those of existing UCN sources; in particular the UCN density would be a factor of $10^{3}$ higher. On the other hand, its parameters are similar to those in most optimistic alternative projects while the price is much lower.

\section{Conflict of Interests}

The authors declare that there is no conflict of interests regarding the publication of this paper.

\section{Acknowledgments}

The authors are sincerely grateful to the staff of the DIN 2Pi instrument, in particular to V. I. Morozov, for their help during this experiment. Also they are sincerely grateful to Y. Shin, C. M. Lavelle, W. Mike Snow, David V. Baxter, Xin Tong, Haiyang Yan, and Mark Leuschner for providing them with the program for calculations of the interaction of neutrons with cold methane.

\section{References}

[1] Y. B. Zel'dovich, "Storage of cold neutrons," Soviet Physics JETP, vol. 9, p. 1389, 1959.

[2] V. I. Luschikov, Pokotilovsky, N. Yu, A. V. Strelkov, and F. L. Shapiro, "Observation of ultracold neutrons," JETP Letters, vol. 9, pp. 23-26, 1969.

[3] J. Butterworth, P. Geltenbort, E. Korobkina et al., "Proceedings of the international workshop on particle physics with slow neutrons, held at the Institut Laue-Langevin, Grenoble, France, October 22-24, 1998. Preface," Nuclear Instruments and Methods in Physics Research A, vol. 440, p. 5, 2000.

[4] V. V. Nesvizhevsky, C. Plonka-Spehr, K. Protasov et al., "Particle physics with slow neutrons. Proceedings of the international workshop on particle physics with slow neutrons, Institut LaueLangevin, Grenoble, France, May 29-31, 2008. Preface," Nuclear Instruments and Methods in Physics Research A, vol. 611, p. 7, 2009.

[5] I. Antoniadis, S. Baessler, O. Bertolami et al., "Workshop GRANIT-2010, 14-19 February 2010, Les Houches, France," Comptes Rendus Physique, vol. 12, no. 8, pp. 703-706, 2011.

[6] R. Golub and J. M. Pendlebury, "Super-thermal sources of ultracold neutrons," Physics Letters A, vol. 53, no. 2, pp. 133-135, 1975.

[7] P. Schmidt-Wellenburg, K. H. Andersen, P. Courtois et al., "Ultracold-neutron infrastructure for the gravitational spectrometer GRANIT," Nuclear Instruments and Methods in Physics Research Section A, vol. 611, pp. 267-271, 2009.

[8] Y. Masuda, T. Kitagaki, K. Hatanaka et al., "Spallation ultracoldneutron production in superfluid helium," Physical Review Letters, vol. 89, no. 28, part 1, Article ID 284801, 2002.

[9] G. Greene, V. Cianciolo, P. Koehler et al., "The fundamental neutron physics beamline at the spallation neutron source," Journal of Research of NIST, vol. 110, pp. 149-152, 2005.
[10] http://nuclear.uwinnipeg.ca/ucn/triumf/post-acot-5-26-8/ucnpost-acot-may08.pdf.

[11] A. P. Serebrov, V. A. Mityukhlyaev, A. A. Zakharov et al., "Project of the ultracold and cold neutron source at the WWR$\mathrm{M}$ reactor with superfluid helium as a moderator," Physics of the Solid State, vol. 52, p. 1034, 2010.

[12] R. Golub, C. Jewell, P. Ageron et al., "Operation of a superthermal ultra-cold neutron source and the storage of ultra-cold neutrons in superfluid Helium," Zeitschrift für Physik B Condensed Matter, vol. 51, no. 3, pp. 187-193, 1983.

[13] Y. Abe and N. Morishima, "Quantitative evaluation of ultracold neutron production and storage in superfluid helium," Nuclear Instruments and Methods in Physics Research Section A, vol. 463, no. 1-2, pp. 293-298, 2001.

[14] P. D. Bangert, M. D. Cooper, and S. K. Lamoreaux, "Enhancement of superthermal ultracold neutron production by trapping cold neutrons," Nuclear Instruments and Methods in Physics Research A, vol. 410, pp. 264-272, 1998.

[15] S. Baessler, A. M. Gagarski, E. V. Lychagin et al., "New methodical developments for GRANIT," Comptes Rendus Physique, vol. 12, no. 8, pp. 729-754, 2011.

[16] V. V. Nesvizhevsky, E. V. Lychaginb, A. Y. Muzychkab, A. V. Strelkovb, G. Pignolc, and K. V. Protasov, "The reflection of very cold neutrons from diamond powder nanoparticles," Nuclear Instruments and Methods in Physics Research A, vol. 595, pp. 631636, 2008.

[17] E. V. Lychagin, A. Y. Muzychka, V. V. Nesvizhevsky, G. Pignol, K. V. Protasov, and A. V. Strelkov, "Storage of very cold neutrons in a trap with nano-structured walls," Physics Letters B, vol. 679, pp. 186-190, 2009.

[18] V. V. Nesvizhevsky, "Interaction of neutrons with nanoparticles," Physics of Atomic Nuclei, vol. 65, no. 3, pp. 400-408, 2002.

[19] S. Grieger, H. Friedrich, K. Guckelsberger et al., "The total neutron scattering cross section of solid methane in phase II," Journal of Chemical Physics, vol. 109, p. 3161, 1998.

[20] Y. Shin, W. Mike Snow, C. Y. Liu, C. M. Lavelle, and D. V. Baxter, "Microscopic model for the neutron dynamic structure factor of solid methane in phase II," Nuclear Instruments and Methods in Physics Research A, vol. 620, no. 2-3, pp. 382-390, 2010.

[21] Y. Shin, C. M. Lavelle, W. M. Snow et al., "Measurements of the neutron brightness from a phase II solid methane moderator at the LENS neutron source," Nuclear Instruments and Methods in Physics Research Section A, vol. 620, no. 2-3, pp. 375-381, 2010.

[22] C. A. Baker, S. N. Balashova, J. Butterworth et al., "Experimental measurement of ultracold neutron production in superfluid ${ }^{4}$ He," Physics Letters A, vol. 308, pp. 67-74, 2003.

[23] E. Korobkina, R. Golub, B. W. Wehring, and A. R. Young, "Production of UCN by downscattering in superfluid He," Physics Letters A, vol. 301, pp. 462-469, 2002.

[24] P. Schmidt-Wellenburg, K. H. Andersena, and O. Zimmera, "Ultra cold neutron production by multiphonon processes in superfluid helium under pressure," Nuclear Instruments and Methods in Physics Research A, vol. 611, pp. 259-262, 2009.

[25] I. V. Kalinin, V. M. Morozov, A. G. Novikov et al., "Characteristics of the DIN-2PI spectrometer with a neutron concentrator," Technical Physics, vol. 59, pp. 307-310, 2014.

[26] Y. G. Dragunov, Y. S. Cherepnin, and V. A. Lukichev, "Conversion of research reactors to low-enrichment fuel," Atomic Energy, vol. 113, no. 1, pp. 39-43, 2012. 

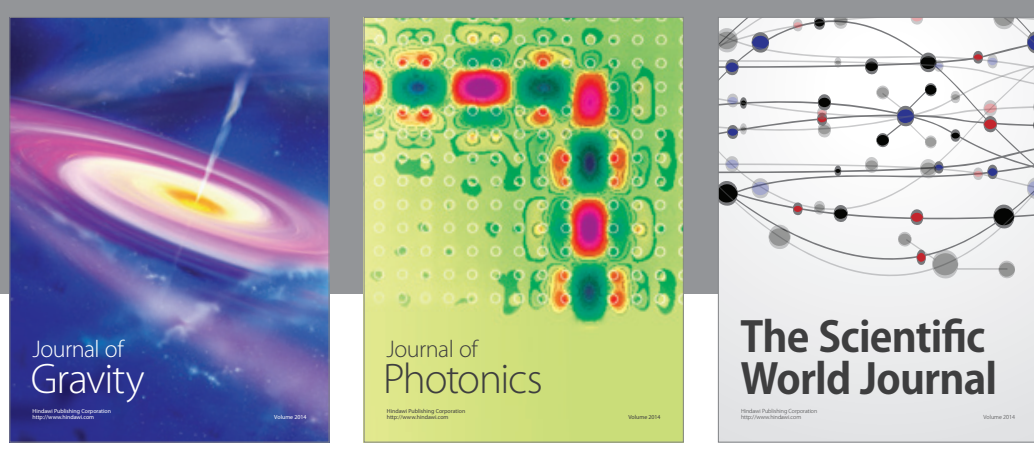

The Scientific World Journal
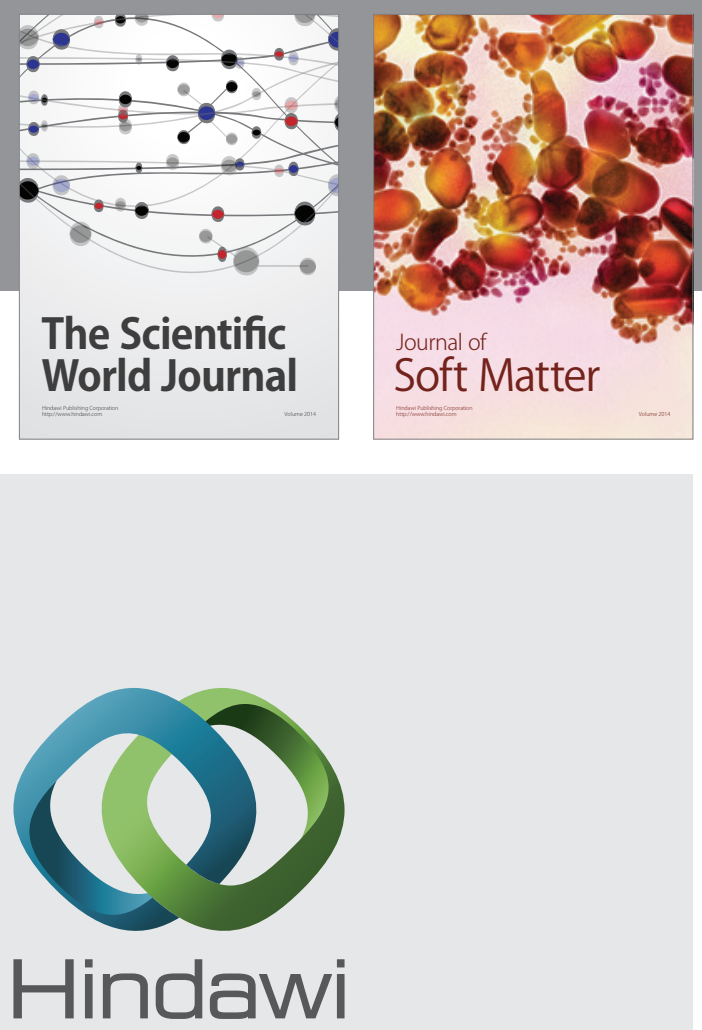

Submit your manuscripts at

http://www.hindawi.com

nternational Journal of

Statistical Mechanics
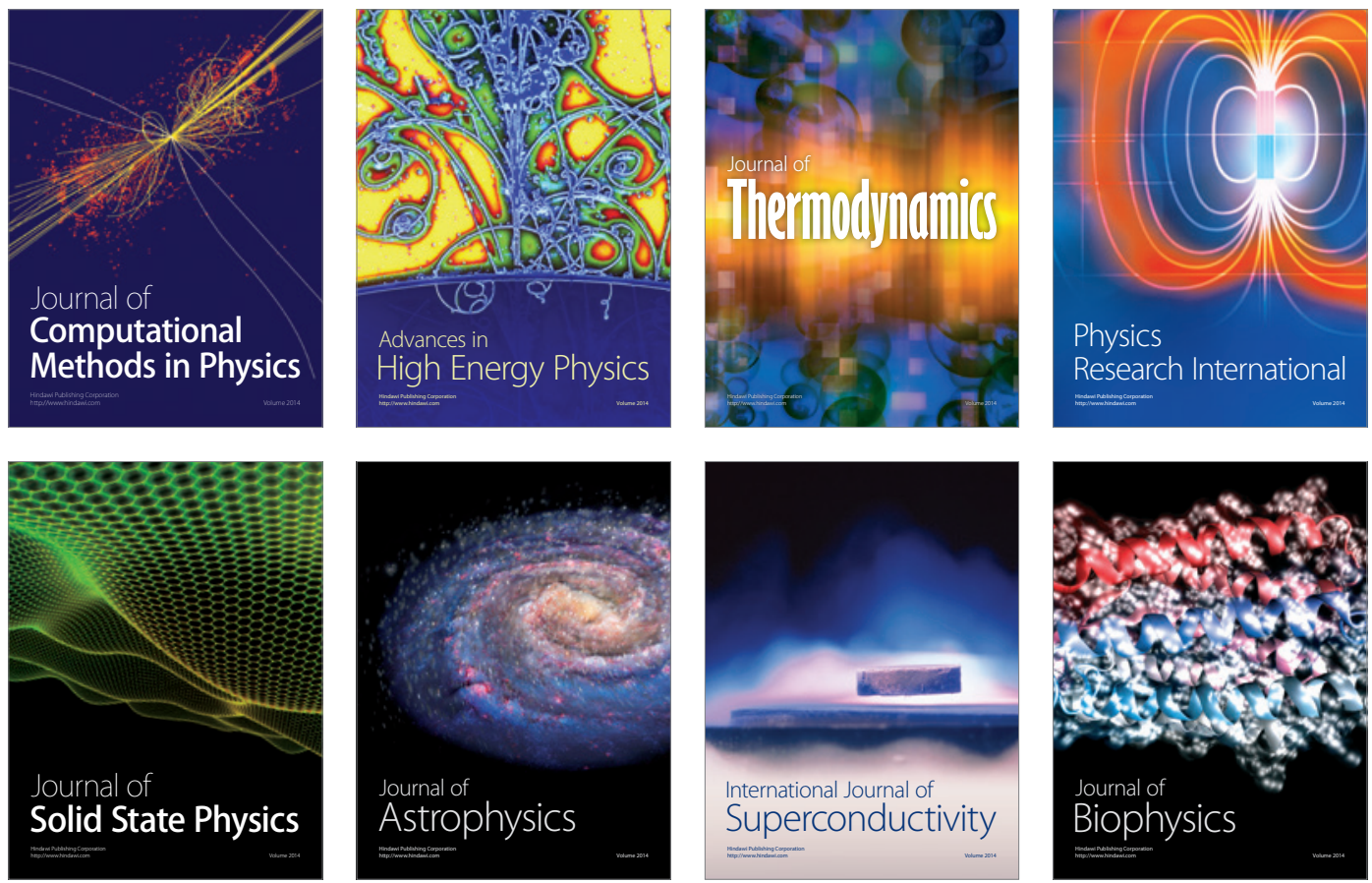
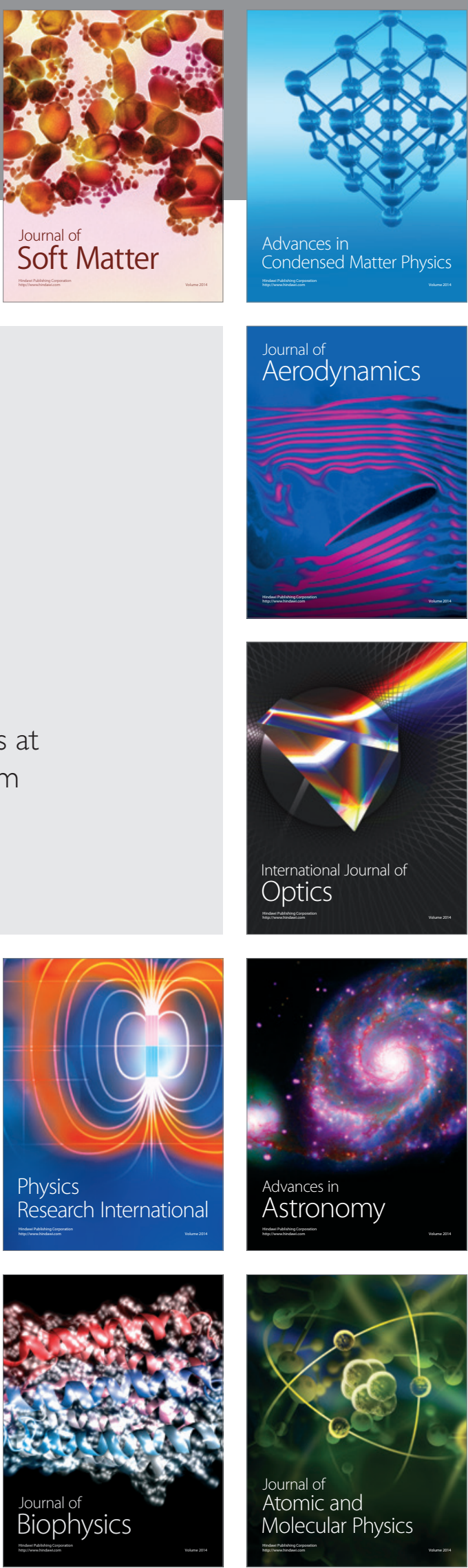